\title{
VULNERABILIDADE, ADAPTAÇÃO E RISCO NO CONTEXTO DAS MUDANÇAS CLIMÁTICAS
}

\author{
vulnerability, adaptation and risk in the context of climate change
}

\author{
Rafael D'Almeida Martins* \\ Leila da Costa Ferreira**
}

\begin{abstract}
Resumo
O objetivo deste artigo é apresentar uma revisão conceitual e analítica da literatura sobre vulnerabilidade, adaptação e risco no âmbito da discussão da variabilidade e mudanças climáticas, que poderá servir para embasar a análise de diferentes perigos climáticos. Após a introdução, o artigo começa com uma distinção entre as perspectivas em relação à vulnerabilidade natural e social e suas interfaces com a noção de risco no contexto geográfico, ressaltando confusões, sobreposições e diferentes estratégias conceituais e metodológicas para abordar essas questões. Esse debate serve de base para introduzir a discussão sobre adaptação e capacidade de adaptação em um contexto mais amplo dado pelas diferentes formas de vulnerabilidade às mudanças do clima. Baseando-se em estudos anteriores disponíveis na literatura internacional sobre o tema, riscos e perigos das mudanças do clima são categorizados e discutidos para posicionar o desafio de localidades brasileiras adaptarem-se a essas mudanças, ressaltando as conexões não só entre capacidade de adaptação e vulnerabilidade, mas também sua dependência em relação ao perigo climático. Adaptação às mudanças climáticas será influenciada por processos externos, sendo necessária uma consideração ampla desses fatores e obstáculos, bem como sua dimensão multiescalar para determinar a capacidade de adaptação da sociedade.
\end{abstract}

Palavras-chave: Vulnerabilidade, Adaptação, Risco, Capacidade

\begin{abstract}
The purpose of this paper is to present a review of conceptual frameworks for studies of vulnerability and adaptation to climate variability and change, generally applicable to a wide range of contexts, systems and hazards. After the introduction, social vulnerability is distinguished from biophysical vulnerability, which is broadly equivalent to the natural hazards concept of risk widespread in geographical studies, highlighting its confusions, assumptions and different conceptual and methodological approaches. This debate serves as an introduction for the discussion about adaptation and adaptive capacity. Based on the international literature, a concise typology of physically-defined hazards is presented in the context of climatic change; the relationship between the vulnerability and adaptive capacity of a human system depends critically on the nature of the hazard to be faced. In addition, the process of adaptation may be inhibited by elements originating outside the system; it is therefore important to consider "external" obstacles to adaptation, and links across scales, when assessing adaptive capacity
\end{abstract}

Keywords: Vulnerability, Adaptation, Risk, Capacity

\section{Resumen}

El objetivo de este trabajo es presentar una revisión conceptual y analítica de la literatura sobre vulnerabilidad, adaptación y riesgo en la discusión de la variabilidad y el cambio climático, que pueden servir para apoyar el análisis de amenazas climáticas diferentes. Después de la introducción, el documento comienza con una distinción entre las perspectivas sobre la vulnerabilidad natural y social y sus interfaces con la noción de riesgo en el contexto geográfico, poniendo de relieve la confusión, superposición y las diferentes estrategias conceptuales y metodológicas para abordar estas cuestiones. Este debate sirve como base para introducir la discusión sobre la adaptación y la adaptabilidad en un contexto más amplio dado por las diferentes formas de vulnerabilidad al cambio climático. Basado en estudios previos disponibles en la literatura internacional sobre el tema, los riesgos y peligros del cambio climático son clasificados y analizados a cabo del reto de las ciudades brasileñas adaptarse a estos cambios, no sólo destacando las conexiones entre la capacidad de adaptación y la vulnerabilidad sino también su dependencia de los riesgos climáticos. Adaptación al cambio climático se verá influido por los procesos externos, que requieren una consideración amplia de eses factores y obstáculos, y para determinar el tamaño y la escala múltiple de la capacidad de adaptación de la sociedad.

Palabras clave: Vulnerabilidad, Adaptación, Riesgo, Capacidad.

(*) Doutorando em Ambiente e Sociedade pela Universidade de Campinas - Rua dos Flamboyants, 155 - Cid. Universitária Zeferino Vaz, CEP: 13083-867, Campinas (SP), Brasil, Tel: (+55 19) 35215103 -rdamartins@gmail.com

(**) Prof ${ }^{a}$. Dra . do Instituto de Filosofia e Ciências Humanas da Universidade de Campinas - Rua dos Flamboyants, 155 - Cid. Universitária Zeferino Vaz, CEP: 13083-867, Campinas (SP), Brasil, Tel: (+55 19) 3521-5103 - leilacf@unicamp.br 


\section{INTRODUÇÃO}

O estudo da vulnerabilidade de populações e ecossistemas às mudanças climáticas e sua variabilidade, bem como suas capacidades de adaptação nesse contexto, é um campo de investigação que ainda pode ser considerado relativamente novo, reunindo pesquisadores com formação em várias disciplinas como Geografia, Climatologia, Biologia, Ecologia e, mais recentemente, também Economia e Ciências Sociais Aplicadas, entre outras áreas do conhecimento (HOGAN, 2007; MARTINS; FERREIRA, 2010). Apesar de muitas vezes buscarem a análise de problemáticas comuns, muitos desses pesquisadores mobilizam um amplo repertório de diferentes perspectivas disciplinares e epistemológicas para desenvolver e aplicar não só modelos teórico-conceituais, como também jargões específicos, para o estudo da vulnerabilidade e adaptação de ecossistemas e grupos sociais em relação aos perigos colocados pelas mudanças do clima .

A emergência das dimensões humanas das mudanças climáticas globais, que exploram problemáticas multifacetadas, multiescalares e complexas, demanda aportes interdisciplinares de análise, que possibilitam não só um diálogo profícuo entre suas diversas abordagens, como também o reconhecimento das várias visões que estão presentes em relação a essas problemáticas de investigação.

Esse é caso para as idéias de vulnerabilidade e adaptação que cada vez mais fazem parte das agendas de pesquisa e dos interesses de governos, agências multilaterais e da sociedade civil em geral (LEICHENKO; O'BRIEN, 2008). O desafio parece residir em desenvolver um olhar capaz de integrar diferentes tradições acadêmicas, teórico-metodológicas e conceituais, de uma forma coerente e flexível, que permita a avaliação não só da vulnerabilidade, como também do potencial de adaptação em uma ampla variedade de contextos, fornecendo elementos que possam ser aproveitados em outros esforços de investigação (BROOKS, 2003).

O volume crescente da literatura sobre vulnerabilidade e adaptação (CUTTER, 1996; ADGER et al., 2002, BURTON et al., 2002; HOGAN; MARANDOLA JR, 2005; IPCC, 2007; PELLING, 2003; 2010) como resultado da aceitação majoritária das mudanças climáticas nos âmbitos internacional e científico, incluindo seus impactos observados e projetados, trouxe acoplado um conjunto de ideias que transpassam as fronteiras estabelecidas pelas disciplinas clássicas. Essa difusão ampliada de termos-chave pode ser ilustrada pela disseminação das noções de vulnerabilidade, sensibilidade, exposição, resiliência, adaptação, capacidade adaptativa, risco, perigo, capacidade de resposta, entre tantas outras, que são cada vez mais presentes não só em círculos acadêmicos, como também na esfera pública e privada do Brasil e do mundo (MARANDOLA JR, 2009).

Apesar da popularidade desses termos, muitas vezes suas relações ainda são pouco claras e o mesmo termo pode ter significados diferentes quando usado em distintos contextos ou por diferentes autores (i.e. CUTTER, 1996; HOGAN; MARANDOLA JR, 2005; 2007; MARANDOLA JR, 2009; MALONE, 2009). Um exemplo dessa afirmação pode ser verificado em relação aos pesquisadores de áreas preocupadas com os perigos e desastres naturais, que tendem a se concentrar na noção de risco (VEYRET, 2007), enquanto que pesquisadores com formação nas ciências sociais aplicadas preferem elaborar suas pesquisas em termos da noção de vulnerabilidade (DOWNING et al., 2001; PELLING, 2003; HOGAN; MARANDOLA JR, 2005).

Essas diferentes perspectivas resultam do fato que cientistas sociais e cientistas naturais referem-se a objetos diferentes quando mobilizam a temática da vulnerabilidade. Enquanto os primeiros tendem a ver a vulnerabilidade como a representação de um contexto histórico determinado por meio de atributos sócio-econômicos e culturais que configuram uma determinada capacidade de resposta a estímulos e mudanças (i.e. CHAMBERS, 1989; MOSER, 1998; ADGER, 1999; DOWNING et al., 2001; PELLING, 2003; EAKIN, 2005; LUERS, 2005; LEICHENKO; O'BRIEN, 2008), os cientistas naturais entendem a vulnerabilidade a partir da probabilidade de ocorrência de danos e perdas que configuram impactos de eventos geofísicos ou hidrometeorológicos sobre um determinado espaço geográfico (NICHOLL et al., 1999; VEYRET, 2007). 
Para contribuir com esse debate, que, apesar de ainda incipiente, vem ganhando novos adeptos no Brasil a partir da expansão de iniciativas como a Rede Clima (CNPq), Instituto Nacional de Ciência e Tecnologia sobre Mudanças do Clima (MCT), Painel Brasileiro de Mudanças Climáticas (PBMC) e Programa de Mudanças Climáticas Globais da Fundação de Amparo à Pesquisa do Estado de São Paulo (FAPESP), este artigo objetiva, de um lado, apresentar uma revisão de aportes teóricos, conceituais e metodológicos das abordagens presentes no interior dos referenciais das dimensões humanas das mudanças climáticas globais para que esses possam ser aplicados de forma consistente em estudos preocupados com a vulnerabilidade e adaptação de localidades brasileiras, sobretudo em razão dos impactos da variabilidade e mudanças climáticas na população e na dinâmica sócio-econômica dessas regiões.

Do outro lado, busca-se colaborar com um esforço continuado de autores brasileiros que há mais de uma década vêm desenvolvendo essa discussão no âmbito de estudos interdisciplinares que se situam na interface entre população, ambiente e sociedade, com ênfase nas áreas urbanas. Autores como Daniel J. Hogan (in memoriam), Francisco de Assis Mendonça e Eduardo Marandola Jr, entre vários outros, são alguns desses nomes que deram contribuições importantes nesse sentido (HOGAN, 2002; HOGAN; MARANDOLA JR, 2005; MARANDOLA JR; HOGAN, 2004a; 2004b; MENDONÇA; LEITÃO, 2008; MARANDOLA JR, 2009; MENDONÇA, 2010; 2011). Internacionalmente, vários trabalhos também possibilitaram um avanço considerável desse campo nas últimas duas décadas, abrindo novos caminhos para essa investigação em distintos contextos (LIVERMAN, 1990; 1994; 2001; CUTTER, 1996; KELLY; ADGER, 2000; KASPERSON; KASPERSON, 2001; CARDONA, 2003; BROOKS, 2003; EAKIN; LUERS, 2006; ADGER, 2006; SMIT; WANDEL, 2006; FÜSSEL, 2007; VEYRET, 2007; PELLING, 2003; 2010).

Nesse sentido, a intenção não é redefinir termos, nem introduzir novas abordagens ou interpretações, que neste momento pouco contribuem para o necessário diálogo destacado anteriormente. Parte-se do pressuposto que, apesar dessas noções estarem consolidas em literaturas específicas, elas adquirem novos significados e interpretações na discussão das mudanças climáticas, tornando-se necessário explorar as noções de vulnerabilidade, adaptação e risco como elas vêm sendo tratadas atualmente, como estratégia para estabelecer conexões e esclarecer algumas das relações entre elas .

Para isso, o texto discute as diferenças entre vulnerabilidade natural e social, seguindo para o debate sobre o risco e como este se relaciona com as noções de vulnerabilidade e perigo mencionadas anteriormente. Diferentes perspectivas são apresentadas e discutidas, assim como as distintas interpretações que o risco adquire quando elevado a uma categoria analítica. A idéia de capacidade adaptativa é explorada, com a ênfase sendo dada na relação desta com o contexto mais amplo de vulnerabilidade. Como pano de fundo dessa discussão aparece a vulnerabilidade atual, futura e potencial de localidades brasileiras e suas correspondentes capacidades de adaptação, que ainda são amplamente desconhecidas e não estudas, dado os vários fatores estruturais que normalmente causam ou agravam a vulnerabilidade de um lugar específico.

\section{AS VÁRIAS FACES DA VULNERABILIDADE: ENTRE A NATUREZA E A SOCIEDADE}

Não é novidade que existem várias concepções distintas para a noção de vulnerabilidade. Para ilustrar essa constatação, Hogan e Marandola Jr (2005) buscaram em Cutter (1996) as quase duas dezenas de diferentes definições para este termo. Adger (1999) também apresentou uma importante resenha de várias abordagens que foram aplicadas em diferentes estudos. Mais recentemente, Marandola Jr (2009) buscou sublinhar a centralidade desse debate para a análise das relações entre população e ambiente. Dessa forma, dado suas múltiplas aplicações, foge do escopo deste trabalho realizar uma análise exaustiva das diferentes idéias-força por trás da vulnerabilidade.

Entretanto, o que há de comum em todas essas concepções é que elas descrevem uma situação de fragilidade de algo ou alguém a partir de um lugar específico, seja ele um grupo populacional, 
uma comunidade, uma cidade, uma região, um país ou um setor da economia em relação a um determinado perigo (ou conjunto de perigos), como no caso das mudanças climáticas. Nesse sentido, o termo perigo passou a ser usado para se referir não só às manifestações físicas de mudanças naturais, como também aquelas induzidas pelas atividades humanas na dinâmica do clima em diferentes escalas.

Tais mudanças podem se manifestar na forma de ressacas de maré, tempestades, secas, inundações, chuvas fortes ou em variações climáticas de longo-prazo. Os chamados perigos climáticos podem ser definidos não só em termos de seus valores absolutos, como também a partir dos desvios em padrões de parâmetros climáticos específicos, como precipitação, temperatura e vento, que podem ser combinados com alterações na magnitude, velocidade, frequência e intensidade desses fenômenos, sendo possível relacionar diretamente os perigos a um ou vários eventos climáticos (BROOKS, 2003; VEYRET, 2007).

De forma geral, os perigos foram historicamente descritos em termos de suas características geofísicas (HEWITT, 1983; VEYRET, 2007). As perdas, danos e eventuais desastres decorrentes desses perigos são mediados por condições gerais, que podem ser analisadas a partir da exposição de uma unidade de análise (e.g. localidade, ecossistema, bacia hidrográfica, comunidade, país) e pelos recursos disponíveis para responder aos impactos desses eventos (BROOKS, 2003; PELLING, 2003; VEYRET, 2007). A partir de um olhar geográfico, as diferentes perspectivas sobre vulnerabilidade em relação às mudanças climáticas tendem a se concentrar em duas grandes categorias, sendo a primeira aquela que enxerga a vulnerabilidade em termos da extensão ou potencial de danos causados por eventos oriundos da dinâmica do clima; já a segunda entende a vulnerabilidade como um estado dinâmico de uma determinada unidade de análise antes que ela sofra com um perigo qualquer (LIVERMAN, 1990; 1994; PELLING, 2003).

A primeira visão surgiu a partir das avaliações de risco e impactos que se popularizaram a partir dos anos 1980, na qual o papel das atividades humanas na mediação das consequências de eventos hidrometeorológicos foi considerado menor ou irrelevante (VEYRET, 2007). Malone (2009) destaca que, tradicionalmente, boa parte dos estudos de impacto das mudanças climáticas, que também foram chamados de estudos da primeira geração (BURTON et al., 2002), segue essa abordagem, onde fatores como o aumento do número de pessoas sob risco de inundação é calculado tendo como base projeções de elevação do nível do mar (i.e. NICHOLLS et al., 1999; MCGRANAHAN et al., 2007).

Nesse caso, o foco de investigação restringe-se em determinar a exposição humana aos diferentes perigos, com pouca consideração em relação à capacidade da sociedade responder aos impactos desses eventos de forma antecipada ou reativa. Dessa forma, a perspectiva dos estudos sobre perigos climáticos e seus impactos entende a vulnerabilidade de pessoas e lugares como sendo uma função das características geofísicas do perigo, da probabilidade ou frequência de sua ocorrência (também considerada por alguns como o risco), da exposição humana ao perigo e, por fim, da sensibilidade da unidade de análise aos impactos desse perigo (CUTTER, 1996; BROOKS, 2003; ADGER, 2006; FÜSSEL, 2007; MALONE, 2009).

Essa forma de conceber os impactos apenas em função do perigo, exposição e sensibilidade, que também pode ser considerada como a análise da vulnerabilidade natural, é ilustrada pela abordagem tradicionalmente dada pelo Intergovernmental Panel on Climate Change (IPCC). Existe um amplo conjunto de trabalhos que, por meio dessa perspectiva, busca métodos robustos para medir e comparar resultados de avaliações de impacto a partir da construção de indicadores baseados em diversas variáveis como custo monetário, mortalidade humana, perdas em serviços ecossistêmicos, etc (BROOKS, 2003; MALONE, 2009).

A segunda perspectiva adota a vulnerabilidade como uma condição ou característica intrínseca de uma determinada unidade de análise (LIVERMAN, 1990). Essa perspectiva ganhou forma a partir de estudos que buscavam investigar os aspectos estruturantes que tornavam determinadas 
comunidades e grupos populacionais específicos suscetíveis a impactos causados por determinados eventos climáticos, sobretudo os episódios dramáticos de seca no continente africano (HEWITT, 1983; WISNER et al., 2004). Nessa formulação, a vulnerabilidade é uma característica inerente ao sistema de análise, algo que está no seu interior, independentemente do perigo climático em questão. Essa abordagem vê a vulnerabilidade como decorrência de características históricas, uma condição interna que se convencionou chamar de vulnerabilidade social (ADGER, 1999; ADGER; KELLY, 1999; KELLY; ADGER, 2000; VEYRET, 2007).

A vulnerabilidade social é determinada por fatores como pobreza, desigualdade, exclusão, insegurança alimentar, indisponibilidade de moradia adequada, entre tantos outros fatores que caracterizam diferentes formas de privação (CHAMBERS, 1989; MOSER, 1998; ADGER; KELLY, 1999; PELLING, 2003; WISNER et al., 2004). Nessa formulação, o resultado negativo ou adverso emerge da interação entre os perigos de um lado e a vulnerabilidade social do outro, produzindo o que Wisner e colegas conceberam como a construção social da vulnerabilidade (WISNER et al., 2004). Tal construção também pode ser determinada a partir dados e indicadores que representem perdas biofísicas, econômicas e dinâmicas sócio-demográficas (i.e. mortalidade populacional, prejuízos em infra-estruturas).

Também se sabe que a natureza da vulnerabilidade social depende do tipo de perigo a que uma determinada comunidade, grupo populacional ou região estiver susceptível. Apesar da vulnerabilidade social não ser nem função da gravidade do perigo, nem da probabilidade de sua ocorrência, alguns atributos poderão tornar essa unidade de análise mais ou menos vulnerável a certos tipos de perigo e menos em relação a outros. Por exemplo, a qualidade e o tipo de moradia pode ser considerado um importante fator determinante da vulnerabilidade de uma comunidade afetada por deslizamentos ou enchentes (PELLING, 2003; VEYRET, 2007).

Assim, embora a vulnerabilidade social não seja função do perigo, ela ainda pode ser contextualizada em termos de um perigo específico, podendo ser sensível não só a fatores genéricos e contextuais como pobreza, desigualdade, acesso a saúde ou acesso e disponibilidade de recursos naturais, como também fatores específicos como a qualidade da moradia, a proximidade de zonas costeiras e de áreas de baixa elevação ou grande inclinação.

Em resumo, a vulnerabilidade natural é uma função da frequência e da probabilidade de ocorrência de um determinado tipo de perigo, enquanto que a vulnerabilidade social não segue o mesmo rigor, uma vez que um perigo numa área desabitada pode não causar danos à população. Da mesma forma, não causam danos aqueles perigos que afetam populações que estão bem preparadas para suportar e responder aos impactos advindos de eventos geofísicos.

Esses são uns dos motivos que tornam a vulnerabilidade social uma categoria analítica interessante a ser considerada, uma que vez que ela favorece a descrição de muitos dos fatores que determinam e influenciam o resultado de um perigo ou evento climático. A vulnerabilidade social incorpora atributos que configuram o resultado de um perigo sobre uma comunidade ou região de forma independente da característica física do perigo a que ela é exposta. Isso inclui as variáveis ambientais e as diversas formas de exposição que também são socialmente determinadas (e.g. segregação espacial), englobando, inclusive, elementos do ambiente físico e como eles se relacionam com os sistemas humanos por meio de fatores como topografia, traçado dos rios e reservatórios de água potável (BROOKS, 2003; PELLING, 2003).

\section{VULNERABILIDADE NO ÂMBITO DE UMA GEOGRAFIA DOS RISCOS}

A ideia de vulnerabilidade natural decorre diretamente da noção de risco e da forma como o mesmo é interpretado pela literatura sobre perigos naturais (VEYRET, 2007). Porém, faz-se necessário uma distinção para evitar confusões dado o grande número de abordagens presentes em distintas formulações. Partindo das sistematizações realizadas por Brooks (2003) e Kelman (2003), o 
quadro 1 ilustra algumas das várias aplicações do termo 'risco' e suas interpretações a partir do que pode ser encontrado em uma amostra de suas múltiplas abordagens. A maioria de suas definições é claramente de natureza probabilística, ou seja, relativas à probabilidade de ocorrência de um perigo que pode desencadear um desastre (ou uma série de eventos com resultados indesejáveis). Também ressaltam a probabilidade da ocorrência de um desastre ou de um resultado indesejado decorrente da ocorrência de um perigo (HEWITT, 1983).

Assim, o resultado da vulnerabilidade também pode ser visto como uma função do risco de ocorrência de um evento (meteorológico) ou da própria vulnerabilidade social. Apesar da polifonia de definições e das diferentes aplicações da terminologia em torno da temática do 'risco', o quadro 1 oferece uma formulação coerente com as definições de risco presentes na literatura (MARANDOLA JR, 2009), sendo a definição do risco apenas como o 'resultado' de um evento geofísico indo de encontro com a definição de vulnerabilidade natural discutida na seção anterior.

Quadro 1 - Diferentes abordagens para 'perigo' e 'risco'

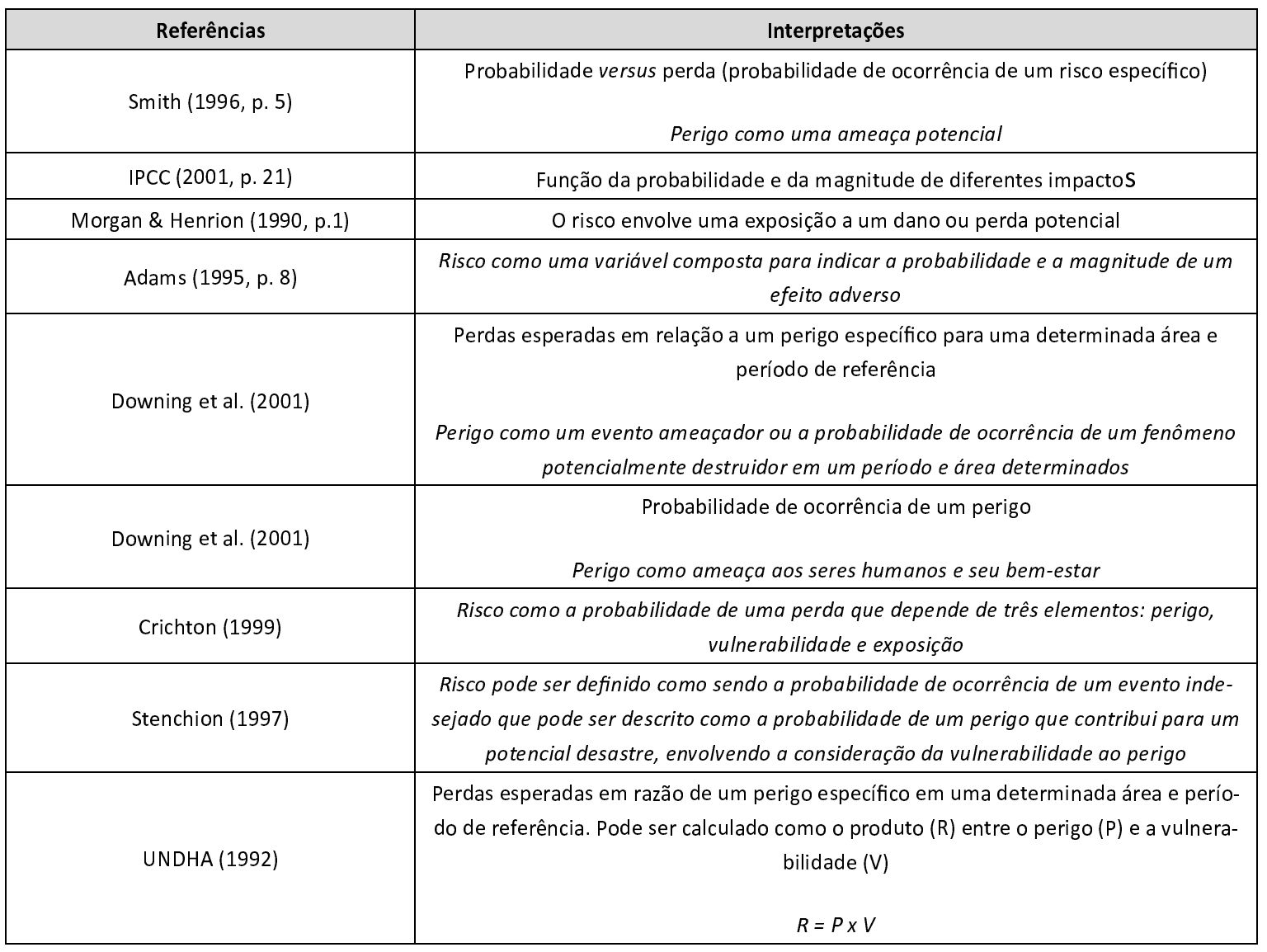

Fonte: Adaptado pelos autores a partir de Brooks (2003) e Kelman (2003).

Se de um lado é nítido que esses vários autores enfatizaram o risco na perspectiva da vulnerabilidade natural, também é interessante notar que pesquisadores investigando as dimensões humanas das mudanças climáticas estão examinando processos correlatos, ou seja, as consequências de eventos hidro-meteorológicos sobre pessoas e lugares, mas aplicando uma outra perspectiva de análise. Ambos os grupos de pesquisa estão interessados em investigar perigos advindos do clima como uma ameaça à sociedade e como algumas características e atributos definidos em termos de suas vulnerabilidade, sensibilidade, resiliência, capacidade de adaptação configuram e influenciam os resultados desses perigos (MALONE, 2009).

Assim, a separação entre vulnerabilidade natural e vulnerabilidade social permite que novas abordagens possam distinguir entre perspectivas que colocam ênfase somente no risco como única categoria analítica daqueles que vêm a vulnerabilidade como um processo dinâmico e dependente 
de vários atributos e vetores de mudança. Dessa maneira, a vulnerabilidade natural continua a ser uma decorrência da abordagem 'fisicalista' que entende 'risco' e 'vulnerabilidade natural' como funções do perigo e da vulnerabilidade social (HEWITT, 1983; WISNER et al., 2004).

A integração de abordagens baseadas no risco e na vulnerabilidade, seja ela natural ou social, pode ser interessante como estratégia metodológica para enfrentar as diferentes ameaças que estão colocadas no presente e no futuro como resultado da variabilidade e mudança climática, além de outros perigos que não estão necessariamente relacionados ao clima. Segundo Kasperson \& Kasperson (2001), torna-se importante avaliar a vulnerabilidade como parte integral de uma cadeia de causalidade que constrói o risco, além de considerar que reduzir a vulnerabilidade da sociedade é uma das medidas mais efetivas para uma estratégia de gestão de risco em relação às mudanças ambientais globais.

Hogan \& Marandola Jr (2005), por sua vez, destacam que a partir da década de 1990 há um aumento no número de perspectivas e definições que reconhecem as múltiplas dimensões da vulnerabilidade e do risco, assinalando que esses não podem ficar restritos a abordagens singulares ou a comunidades científicas específicas. Citando o trabalho seminal de Blaikie et al. (1994) [WISNER et al., 2004], os autores mostram que essa busca reside em evitar uma separação artificial entre o risco - como a probabilidade de que pessoas venham a sofrer por conta de perigos naturais - do risco a que as pessoas estão expostas cotidianamente na sociedade, unificando de certa maneira 'riscos naturais' a 'riscos sociais' (HOGAN; MARANDOLA JR, 2005, p. 461).

Essa evolução na forma de conceber a 'vulnerabilidade' e o 'risco' caminhou na direção de incluir, além das dinâmicas biofísicas, também as dimensões sociais, econômicas, políticas e demográficas que são elementos centrais na estrutura causal que determina aqueles que são vulneráveis aos perigos (eventos climáticos ou meteorológicos) (KASPERSON; KASPERSON, 2001). Neste caso, a 'vulnerabilidade' e o 'risco' são as categorias analíticas que permitem uma reflexão dinâmica que vai além do evento em si ou do lugar como espaço geográfico, ou seja, ultrapassando suas características físicas e morfológicas, para englobar os demais processos que configuram e definem uma sociedade.

Ainda segundo Hogan \& Marandola Jr (2005), essa perspectiva analítica mais ampla e elaborada possibilita uma discussão enriquecida das questões referentes às escalas de análise e um diálogo mais próximo dessas abordagens com a teoria da 'risk society' (e.g. BECK, 1992), que aparenta estar ausente no debate dos estudos geográficos sobre risco, perigo e vulnerabilidade (MARANDOLA JR; HOGAN, 2004a; 2004b; HOGAN; MARANDOLA JR, 2005; VEYRET, 2007; PELLING, 2010). Vale deixar claro que essa formulação - teoria da risk society - recebeu críticas importantes daqueles que analisam situações de risco e vulnerabilidade no contexto dos países pobres e emergentes, uma vez que esses enxergaram na formulação teórica da 'sociedade de risco' um viés notadamente dado pelo contexto das sociedades ocidentais modernas (i.e. WISNER et al., 2004). Nesse sentido, no contexto desses países pobres, os paradigmas da 'modernização reflexiva' (e.g. GIDDENS, 1990; BECK, 1992) e 'modernização ecológica' (i.e. SPAARGAREN et al., 2000) ainda não podem ser considerados plenamente estabelecidos, apesar de existirem pontos de contato entre essas duas concepções teórico-metodológicas para análise das causas ou raízes da vulnerabilidade (HOGAN; MARANDOLA JR, 2005).

A inserção da noção de vulnerabilidade social no contexto da abordagem de risco e de vulnerabilidade natural pode não só caminhar para uma menor confusão associada às definições de vulnerabilidade, como também facilitar uma melhor comunicação entre pesquisadores com diferentes formações disciplinares, melhorando as perspectivas de trabalhos realmente colaborativos e interdisciplinares para compreensão dos velhos e novos desafios colocados pela variabilidade e mudança do clima no século XXI (PELLING, 2010).

Com efeito, espera-se que a discussão acima sirva como um alerta para um uso mais cuidadoso e criterioso dessas várias abordagens existentes, ao invés de uma busca sem fim para a redefinição 
contínua de termos-chave como vulnerabilidade e risco. Embora se reconheça que diferentes contextos exigem diferentes abordagens, é essencial que investigadores que trabalham no mesmo campo utilizem não só uma linguagem comum, como também se faz necessário que usem essas abordagens como forma de reconciliar diferenças e unir os esforços necessários para a construção do que Cutter (2003) chamou de uma 'ciência da vulnerabilidade' (HOGAN; MARANDOLA JR, 2005).

\section{CAPACIDADE, ADAPTAÇÃO E VULNERABILIDADE: PARA ALÉM DO RISCO}

A discussão acima buscou dar as bases para a elaboração de um marco conceitual e analítico que pudesse de alguma forma diferenciar entre as variadas abordagens que estão colocadas para a discussão, análise e avaliação da vulnerabilidade natural, do risco e da vulnerabilidade social. Buscou-se destacar diferenças e articular um diálogo para que seja possível não só um avanço dessa área, como também uma interpretação mais crítica dos enormes esforços de sistematização do estado da arte da ciência em relação aos temas ambientais globais, como as realizadas pelo IPCC e Millennium Ecosystem Assessment (MEA). Apesar dessas iniciativas terem alcançado status de referências importantes para o debate e ação política em relação a esses temas, seus relatórios apresentam capítulos cujos usos dos termos vulnerabilidade, adaptação e risco são confusos e, muitas vezes, até contraditórios, ao referir-se aos perigos da variabilidade do clima ou a sensibilidade e vulnerabilidade de sistemas sócio-ecológicos (BROOKS, 2003).

Nesse momento, faz-se necessário abordar a questão referente à capacidade adaptativa da sociedade ou de um ecossistema em relação ao risco e a vulnerabilidade. Na discussão das mudanças climáticas, a literatura define a capacidade adaptativa como sendo a capacidade que um sistema apresenta para modificar ou alterar suas características gerais como uma resposta antecipada a estímulos climáticos, podendo eles ser atuais ou futuros (IPCC, 2001; BURTON et al., 2002; ADGER et al., 2003; SMIT; WANDEL, 2006).

De acordo com essa abordagem, a idéia de adaptação é utilizada para indicar ajustes tanto nos padrões de funcionamento de um determinado sistema, como nas suas características, que melhoram ou aumentam a sua capacidade de responder aos mais variados estímulos climáticos (PELLING, 2010). No caso de comunidades, ou mesmo cidades, imaginando que os níveis de perigo ao longo do tempo serão constantes, medidas de adaptação podem possibilitar a redução dos riscos associados a esses perigos por meio da redução da vulnerabilidade da população e das infra-estruturas (WILBANKS et al., 2007).

Assim, cidades que enfrentam níveis de perigo crescentes, demandarão medidas de adaptação para manter os padrões atuais de risco (PELLING, 2003). Atualmente, em localidades onde os riscos já são altos e considerando o cenário de perigos crescentes dado pelas projeções de mudança climática (IPCC, 2001; 2007), será necessário um esforço ainda maior na direção da adaptação (PELLING, 2010). Assim, se a frequência e a intensidade dos perigos aumentam (IPCC, 2007; WILBANKS et al., 2007), a cidade passará a enfrentar maior risco, mesmo que reduções na vulnerabilidade social tenham sido conseguidas por meio de estratégias diversas de adaptação no passado e no presente.

Portanto, uma consequência direta das medidas de adaptação é que elas não só podem, como deveriam reduzir a vulnerabilidade social. No caso específico das mudanças climáticas decorrentes de atividades humanas, a única forma sustentável de reduzir o risco no longo prazo é por meio da utilização de estratégias combinadas de mitigação - cortes nas emissões de gases de efeito estufa (GEE) - e de adaptação (PARRY et al., 2008; PARRY, 2009; PELLING, 2010).

\section{Vulnerabilidade e adaptação no contexto de perigos específicos}

Parece óbvio que não faz sentido falar em capacidade adaptativa ou em vulnerabilidade de uma localidade sem especificar o perigo a que ela deva se adaptar. Dessa forma, faz-se necessário o reconhecimento de que tanto a vulnerabilidade natural e o risco, assim como a vulnerabilidade 
social e a capacidade de adaptação, são específicas não só a um determinado contexto, como também a um território e sua dimensão temporal. No caso das mudanças climáticas, são vários os tipos de perigos ou eventos climáticos que poderão ser exacerbados ou introduzidos em diferentes escalas espaciais e temporais, exigindo múltiplas respostas em termos de adaptação. Assim, uma localidade pode ter capacidade para adaptar-se a certos tipos de perigo, mas a outros provavelmente não, requerendo tempo e investimentos no sentido de aumentar sua capacidade adaptativa em relação aos novos perigos que poderão estar colocados. Tendo em vista as mudanças do clima, utilizamos três grandes categorias que foram propostas por Brooks (2003) para iluminar esse debate.

Categoria 1: Repetição de perigos variados, incluindo eventos extremos decorrentes da variabilidade do clima, como no caso de fenômenos como tempestades, secas ou ressacas de maré;

Categoria 2: Perigos contínuos ao longo do tempo, como aumentos de temperaturas médias, elevação do nível do mar e a diminuição dos níveis de precipitação ao longo de décadas;

Categoria 3: Perigos variados únicos, como no caso de mudanças abruptas do clima ou em padrões associados a correntes marítimas, cuja mudança pode perdurar por séculos, talvez milênios.

Um fato que merece destaque por sua relevância é que ter capacidade adaptativa não garante que a adaptação acontecerá, uma vez que essa depende de tempo, aprendizado e uma série de outros condicionantes bastante subjetivos (BURTON et al., 2002, SMIT; WANDEL, 2006). Basta considerar as perdas econômicas e de vidas humanas causadas por eventos extremos recentes como ciclones tropicais e ondas de calor na América do Norte e Europa, regiões industrializadas com grande capacidade de adaptação. Nesse sentido, a capacidade adaptativa indica um potencial de adaptação, mas não a adaptação per se. Por exemplo, um nível elevado de capacidade adaptativa no momento pode indicar uma baixa vulnerabilidade social aos perigos climáticos no futuro, mas não garante que as adaptações necessárias no futuro serão feitas. Assim, para transformar a capacidade adaptativa em adaptações de facto é necessário uma série de fatores que podem apoiar ou obstruir essas iniciativas (PELLING, 2010).

Em outras palavras, a capacidade adaptativa é um fator determinante da vulnerabilidade à Categoria 2 de perigos. Assim, a experiência de um perigo hoje não depende diretamente da capacidade de empreender medidas no futuro. Nesse caso, o mais interessante seria analisar as medidas de adaptação que já estão implementadas no presente, que foram determinadas por uma capacidade adaptativa do passado para enfrentar os perigos sofridos no momento (BROOKS, 2003; SMIT; WANDEL, 2006). No entanto, a vulnerabilidade de um sistema a mudanças graduais, de longo-prazo, será função de sua capacidade de se adaptar de forma incremental e responsável, enquanto que a sua vulnerabilidade aos perigos específicos que poderão ocorrer no futuro será função de sua capacidade de prever e antecipar-se a esses perigos por meio de estratégias adequadas de adaptação.

Em termos das mudanças climáticas, é difícil definir de maneira precisa em que medida a sociedade poderá diminuir a vulnerabilidade natural e social de uma determinada localidade, uma vez que essa depende, por exemplo, do prazo disponível para a identificação, avaliação e adoção de medidas de adaptação necessárias, bem como de estabelecer uma capacidade adaptativa no momento. Um caso interessante e de extrema relevância é a questão da elevação do nível do mar. O IPCC projetou uma elevação de $19 \mathrm{~cm}$ a $59 \mathrm{~cm}$ até o final do século XXI . No Brasil, estima-se que o nível do mar venha aumentando cerca de $40 \mathrm{~cm}$ por século de acordo com dados da pesquisa coordenada pelo Prof. Afrânio Mesquita (IO/USP) e uma série de impactos, sobretudo em termos de erosão da costa brasileira, já podem ser observados em muitas localidades da zona costeira brasileira . Assim, o risco colocado por esse perigo dependerá não só da taxa de elevação do nível 
do mar, mas também da vulnerabilidade de cada região afetada, além do tempo necessário para a adaptação e a capacidade dessas regiões em promover as medidas necessárias de forma planejada.

\section{Capacidade adaptativa e vulnerabilidade atual e futura}

Outra forma de abordar o tempo necessário para a adaptação é pensar em termos da vulnerabilidade atual e futura. A vulnerabilidade atual, determinada pelas adaptações do passado e a atual capacidade adaptativa, que também podem ser entendidas como o estoque de recursos e conhecimentos que poderão ser mobilizados para implementar ações futuras, fornece um ponto de referência que pode ser interessante para pensar as cidades e os impactos das mudanças climáticas e como essas poderão empreender as medidas necessárias no futuro. As ações futuras serão dependentes tanto da capacidade adaptativa que existe no momento como a forma pela qual essa capacidade será empregada para a realização da adaptação (BROOKS, 2003).

Num dado momento, podemos analisar uma localidade em termos de sua vulnerabilidade atual em relação a um ou mais perigos e discutir qual sua vulnerabilidade potencial caso a frequência ou intensidade desses perigos mudem no futuro. Pode-se dizer que essa vulnerabilidade potencial, marcada por um período específico no futuro, será resultado da mobilização de sua capacidade adaptativa do momento e do grau de adaptação alcançado como estratégia para antecipar os impactos desses perigos. Assim, mudanças em aspectos sociais, econômicos, políticos, institucionais e culturais podem aumentar ou diminuir a capacidade adaptativa no presente e influenciar positiva ou negativamente a adaptação no futuro, caso essas medidas sejam empreendidas (BROOKS, 2003).

Em linhas gerais, pode-se dizer que a vulnerabilidade social é fortemente marcada tanto pelas vias de desenvolvimento de uma determinada localidade quanto pelo tipo e padrão de perigos que ela está exposta (LEICHENKO \& O'BRIEN, 2008; PELLING, 2010). Também é importante notar que esses processos podem apresentar vários feedbacks. Assim, mesmo que num primeiro momento um determinado perigo não tenha tido impacto sobre uma região, as consequências desse impacto no meio físico podem impactar a sociedade num segundo momento. Por exemplo, as consequências de um perigo podem a priori não causar impactos em determinada comunidade, porém os danos desse impacto no meio ambiente em termos da destruição de estoques de recursos naturais poderão impactar a comunidade num segundo momento, exacerbando situações de dificuldade e escassez com impactos sobre os níveis de pobreza, exclusão ou desigualdade (LEICHENKO; O'BRIEN, 2008).

\section{NOVOS CAMINHOS: determinar a capacidade de adaptação}

A discussão acima enfocou a relação entre a capacidade adaptativa e a vulnerabilidade, analisando a noção de capacidade adaptativa em termos amplos. No entanto, caso os objetivos do pesquisador sejam avaliar a capacidade adaptativa existente é necessário compreender os elementos que fazem parte de sua construção e de que maneira essa capacidade adaptativa pode ser traduzida em medidas concretas de adaptação.

Em outras palavras, deve-se entender o processo de adaptação, considerando as características da unidade de análise que se deseja adaptar. Assim, as formas pelas quais uma comunidade de pescadores se adapta às mudanças climáticas no nível local será diferente das estratégias de adaptação que o Brasil poderá implementar no seu setor agrícola por exemplo. No primeiro caso, a adaptação será determinada por fatores tais como nível de educação, acesso à saúde, acesso à informação, tecnologia, disponibilidade de recursos financeiros, além da presença ou não de conflitos na comunidade e a existência de redes de apoio que muitas vezes são vistas em termos de capital social (ADGER, 2003). No caso do setor agrícola brasileiro, a adaptação vai depender das relações entre o governo federal com o setor privado e a sociedade civil, o ambiente regulatório e o marco legal, além da eficácia das instituições de Estado, do setor privado e o volume de recursos disponíveis para essas ações (BROOKS, 2003; ADGER, 2003; SMIT; WANDEL, 2006). 
Esses exemplos buscam ilustrar os fatores que são fundamentais para que medidas de adaptação possam ser levadas adiante ou não, dependendo de processos que acontecem em diferentes escalas. Assim, diferentes unidades de análise são caracterizadas por diferentes escalas e as diferentes unidades podem interagir umas com as outras. Assim, processos operando dentro de uma unidade de análise poderão afetar direta ou indiretamente outra unidade e assim por diante (LEICHENKO; O'BRIEN, 2008). Exemplos de tais conexões que também são chamadas de trans-escalares incluem intervenções no mercado global que podem alterar o preço de uma commodity, impactando o pequeno produtor no nível local, que ao ter menos recursos disponíveis, deixa de lado medidas preventivas para antecipar perigos climáticos específicos como a seca, aumentando sua vulnerabilidade social na ocorrência do perigo (BROOKS, 2003; LEICHENKO; O'BRIEN, 2008; PELLING, 2010).

Dessa forma, as unidades de análise não são fechadas, nem herméticas; tampouco se pode avaliar a capacidade adaptativa sem considerar o papel dos vários obstáculos à adaptação, que podem ser determinados por processos que operam fora da própria unidade de análise em questão. Esse fato é de suma importância, uma vez que tanto os fatores endógenos quanto exógenos influenciam o processo de adaptação, ressaltando mais uma vez as complexas interações entre os níveis de análise e as escalas de ação. Dessa maneira, é muito difícil operacionalizar a pesquisa de adaptação em termos práticos considerando todas as possíveis interações e feedbacks no interior do sistema, levando em consideração todas as possíveis unidades de análise.

Do outro lado, em termos conceituais e metodológicos, manter o olhar atento não só nos processos que ocorrem nos níveis reduzidos de interação (i.e. local), mas também considerando contextos mais amplos de ordem social, econômica, política e ambiental pode ser uma estratégia importante para captar vetores diretos e indiretos de vulnerabilidade e de capacidade adaptativa. Uma abordagem mais ampla pode, assim, auxiliar na determinação das verdadeiras causas da vulnerabilidade (HEWITT, 1983; PELLING, 2003; 2010).

Nesse sentido, Brooks (2003) faz um alerta chamando a atenção para o fato que existe um temor de que a idéia de capacidade adaptativa seja utilizada da mesma forma que o conceito de capital social vem sendo aplicado por organismos multilaterais (e.g. Banco Mundial e Programa das Nações Unidas para o Desenvolvimento - PNUD). Trata-se de negligenciar as arenas de poder e conflito de tal maneira que as ações empreendidas não conseguem alcançar a dimensão estrutural dos processos que provocam a pobreza, a desigualdade, a exclusão e a vulnerabilidade (BROOKS, 2003, p. 12).

Em resumo, os processos que determinam se a sociedade será capaz de se adaptar acontecem em uma variedade de escalas. Ao adotar como pressuposto que a capacidade adaptativa é algo inerente à uma unidade de análise, a ênfase será dada nos processos e nas interações ocorrendo em nível reduzido, que é delimitado pelas relações no interior da unidade de análise, com pouca ou nenhuma consideração sendo dada a processos de larga-escala, como a globalização ou as grandes negociações internacionais do clima no caso das mudanças climáticas. Brooks (2003) ressalta que a questão da escala nos leva a pensar com mais cuidado sobre a própria definição de capacidade adaptativa que vem sendo disseminada.

A abordagem de capacidade adaptativa deve buscar englobar a maior quantidade de processos possíveis que sejam capazes de explicar os 'por quês' de que a adaptação poderá acontecer ou não acontecer. Para isso é necessário considerar os distintos contextos ambientais, econômicos, sociais, políticos e institucionais, bem como ter uma perspectiva histórica, uma vez que esses processos dependem das trajetórias de desenvolvimento e das maneiras pelas quais se constroem as situações vulnerabilidade ao longo do espaço e do tempo (HEWITT, 1983; WISNER et al., 2004; PELLING, 2010). 


\section{CONSIDERAÇÕES FINAIS}

Este trabalho buscou articular o diálogo entre as noções de vulnerabilidade, adaptação, capacidade adaptativa e risco a partir dos seus diferentes debates e perspectivas de análise. Em primeiro lugar, a vulnerabilidade foi apresentada em termos de suas duas principais linhagens na literatura, quais sejam, a vulnerabilidade natural ou biofísica que tem origem nos estudos sobre os perigos e desastres naturais; e a vulnerabilidade social, marcada pela economia política de aspectos sociais e ambientais que configuram uma situação de privação de meios e recursos para responder a estímulos diversos. Por meio dessa discussão, uma articulação foi feita como forma de contribuir com uma interpretação mais clara não só da terminologia que pode ser usada por diferentes pesquisadores interessados em investigar as dimensões humanas e sociais das mudanças climáticas globais, como também destacar os desafios que estão colocados para essas iniciativas.

Ao reconhecer as convergências entre a vulnerabilidade natural e a noção de risco no contexto de uma geografia dos riscos ambientais, coloca-se o estudo da vulnerabilidade social num quadro mais amplo de redução da pobreza e gestão de risco. Dentro desse quadro, o risco para a sociedade será dado por um tipo específico de perigo a ser definido em termos da sua magnitude, intensidade e freqüência, além das formas pelas quais suas consequências serão mediadas pela própria vulnerabilidade social de uma localidade ou população. Por sua vez, a capacidade adaptativa dessa localidade ou comunidade representa o potencial que esses apresentam para reduzir sua vulnerabilidade social e, portanto, englobam um conjunto amplo de recursos e meios que podem ser mobilizados para minimizar os riscos associados a um determinado perigo.

Embora sejam muitos os fatores que podem determinar essa capacidade para se adaptar aos vários riscos existentes e projetados, alguns aspectos da capacidade adaptativa dependem do tipo de perigo a ser experimentado. A natureza dos perigos enfrentados por uma localidade ou comunidade e as escalas temporais associadas a eles serão fundamentais para determinar o conjunto da capacidade adaptativa e as estratégias adequadas de adaptação.

Apesar de várias décadas de estudos sobre perigos climáticos e seus efeitos sobre lugares e populações específicas, estudos que buscam articular um olhar que reconhece a vulnerabilidade social e a capacidade de resposta e adaptação da sociedade são mais recentes e ainda carecem de maior aprofundamento conceitual e metodológico, bem como de maior relevância empírica. Estudos direcionados a discutir essas e outras questões no contexto particular das mudanças climáticas de natureza humana são ainda mais recentes, sendo praticamente inexistentes no país. Internacionalmente, esses estudos também podem ser considerados em sua infância. Apesar da urgência de iniciativas nesse sentido e da carência de aportes mais aprofundados para análise da possível realidade futura do país, é necessário levar em conta as mazelas brasileiras em termos sociais e ambientais como forma de evitar adaptações ineficazes ou que reforçam situações de injustiça e exclusão.

\section{AGRADECIMENTOS}

Os autores agradecem o auxílio financeiro da Coordenação de Aperfeiçoamento de Pessoal de Nível Superior (CAPES) e da Fundação de Amparo à Pesquisa do Estado de São Paulo (FAPESP), assim como a hospitalidade do Department of Environmental Policy Analysis (EPA), Institute for Environmental Studies (IVM), Vrije Universiteit Amsterdam, Holanda, onde parte desta pesquisa foi realizada. Também são gratos aos comentários recebidos dos Professores Wagner Costa Ribeiro (FFLCH-USP) e Carlos Alfredo Joly (IB/UNICAMP e Ministério de Ciência \& Tecnologia - MCT).

\section{REFERÊNCIA BIBLIOGRÁFICA}

ADAMS, J. Risk. London: University College London Press, 1995.

ADGER, W.N. Social Aspects of Adaptive Capacity. In: SMITH, J.B.; KLEIN, R.J.T.; HUQ, S. Climate 
Change, Adaptive Capacity and Development. London: Imperial College Press, 2003.

ADGER, W.N. Social Vulnerability to Climate Change and Extremes in Coastal Vietnam. World Development, v. 27, n. 2, p. 249-269, 1999.

ADGER, W.N. Vulnerability. Global Environmental Change, v. 16, n. 3, p. 268-281, 2006.

ADGER, W.N.; HUQ, S.; BROWN, K.; CONWAY, D.; HULME, M. Adaptation to climate change: setting the agenda for development policy and research. Tyndall Centre for Climate Change Research Working Paper 16, 2002.

ADGER, W.N.; KELLY, P.M. Social vulnerability to climate change and the architecture of entitlements. Mitigation and Adaptation Strategies for Global Change, v. 4, p. 253-266, 1999.

BECK, U. Risk Society: Towards a New Modernity. London: SAGE, 1992.

BLAIKIE, P.M.; CANNON, T.; DAVIS, I. WISNER, B. At Risk: Natural Hazards, People's Vulnerability , and Disasters. London: Routledge, 1994.

BROOKS, N. Vulnerability, risk and adaptation: a conceptual framework. Tyndall Centre for Climate Change Working Paper 38, 2003.

BURTON, I.; HUQ, S.; LIM, B.; PILIFOSOVA, O.; SCHIPPER, E.L. From impacts assessment to adaptation priorities the shaping of adaptation policies. Climate Policy, v. 2, p. 145-159, 2002.

CARDONA, O.D. The need to rethinking the concepts of vulnerability and risk from a holistic perspective: a necessary review and criticism for effective risk management. In: BANKOFF, G.; FRERKS, G.; HILHORST, D. (Orgs.). Mapping Vulnerability: Disasters, Development and People. London: Earthscan, 2003.

CHAMBERS, R. Editorial introduction: Vulnerability, coping and policy. IDS Bulletin, v. 20, n. 2, p. 7, 1989. CRICHTON, D. The risk triangle. In: Ingleton, J. (Org.). Natural Disaster Management. London: Tudor Rose, p. 102-103, 1999.

CUTTER, S.L. The vulnerability of science and the science of vulnerability. Annals of the Association of Geographers, v. 93, p. 1-12, 2003.

CUTTER, S.L. Vulnerability to Environmental Hazards. Progress in Human Geography, v. 20, n. 4, p. 529-539, 1996.

DOWNING, T.E.; BUTTERFIELD, R.; COHEN, S.; HUQ, S.; MOSS, R.; RAHMAN, A.; SOKONA, Y.; STEPHEN, L. Vulnerability Indices: Climate Change Impacts and Adaptation. UNEP Policy Papers, Nairobi, 2001.

EAKIN, H. Institutional change, climate risk and rural vulnerability. Cases from central Mexico. World Development, v. 33, n. 1, p. 1923-1939, 2005.

EAKIN, H.; LUERS, A. Assessing the vulnerability of social-environmental systems. Annual Review of Environment and Resources, v.31, p. 365-394, 2006.

FERREIRA, L.C.; BARBOSA, S.R.C.S.; HOEFEL, J.L.M.; GUIMARAES, R.P.; FLORIANI, D.; TAVOLARO, S.B.F. Environmental Issues, Interdisciplinarity, Social Theory and Intellectual Production in Latin America. Ambiente \& Sociedade, v. 9, n. 2, p. 9-24, 2006.

FÜSSEL, H.M. Vulnerability: A Generally Applicable Conceptual Framework for Climate Change Research. Global Environmental Change, v. 17, n. 2, p. 155-167, 2007.

GIDDENS, A. The Consequences of Modernity. Cambridge: Polity Press, 1990.

HOGAN, D.J. Human dimensions of global environmental change. Ambiente \& Sociedade, v.10, n.2, p. 161-166, 2007.

HOGAN, D.J. Movilidad poblacional, sustentabilidad ambiental y vulnerabilidad social: una perspectiva latino-americana. In: LEFF, E.; EZCURRA, E.; PISANTY, I.; LANKAO, P.R. (Orgs.) La transición hacia el desarrollo sustentable. Perspectivas de América Latina y el Caribe. México: INE-SEMARNAT, UAM, PNUMA, 2002.

HEWITT, K. Interpretations of Calamity: From the Viewpoint of Human Ecology (The Risks \& Hazards Series, 1). London: Allen \& Unwin, 1983.

HOGAN, D.J.; MARANDOLA JR, E. Towards an interdisciplinary conceptualisation of vulnerability. Population, Space and Place, v. 11, n. 6, p. 455-471, 2005. 
HOGAN, D.J.; MARANDOLA JR, E. Vulnerabilidade e perigos naturais nos estudos de população e ambiente. In: HOGAN, D.J. (Org.) Dinâmica populacional e mudança ambiental: cenários para o desenvolvimento brasileiro. 1 ed. Campinas: NEPO/UNFPA, 2007, p. 41-60.

IPCC (Intergovernmental Panel on Climate Change). Climate Change 2001. Synthesis Report. Cambridge: Cambridge University Press, 2001.

IPCC (Intergovernmental Panel on Climate Change). Climate Change 2007. Synthesis Report. Cambridge: Cambridge University Press, 2007.

KASPERSON, J.X.; KASPERSON, R.E. International Workshop on Vulnerability and Global Environmental Change. Stockholm: SEI, 2001.

KELLY, P.M.; ADGER, W.N. Theory and Practice in Assessing Vulnerability to Climate Change and Facilitating Adaptation. Climatic Change, v. 47, n. 4, 2000.

KELMAN, I. Defining Risk. Flood Risk Net Newsletter, Issue 2, 2003.

LEICHENKO, R.M.; O'BRIEN, K.L. Environmental Change and Globalization: Double Exposures. New York: Oxford University Press, 2008.

LIVERMAN, D. Vulnerability to global environmental change. In: KASPERSON, R.E. et al. (Orgs.). Understanding Global Environmental Change: The Contributions of Risks Analysis and Management. The Earth Transformed Programme, Clark University, Worcester MA, 1990, p. 27-44.

LIVERMAN, D.M. Vulnerability to global environmental change. In: CUTTER, S.L. (Org.) Environmental Risks and Hazards. London: Prentice-Hall, 1994.

LUERS, A.L. The surface of vulnerability: An analytical framework for examining environmental change. Global Environmental Change, v. 15, p. 214-223, 2005.

MALONE, EL. Vulnerability and Resilience in the Face of Climate Change: Current Research and Needs for Population Information. Population Action International. Battelle: The Business of Innovation. PNWD-4087, 2009.

MARANDOLA JR, E. Tangenciando a vulnerabilidade. In: HOGAN, D.J.; MARANDOLA JR, E. (Orgs.). População e mudança climática: Dimensões humanas das mudanças ambientais globais. Campinas: Ed. UNICAMP, 2009, p. 29-52.

MARANDOLA JR, E.; HOGAN, D.J. Natural hazards: o estudo geográfico dos riscos e perigos. Ambiente \& Sociedade, v. 7, p. 95-109, 2004a.

MARANDOLA JR, E.; HOGAN, D.J. O risco em perspectiva: tendências e abordagens. Geosul, v. 19, n. 38, p. 25-58.

MARTINS, R.D.A.; FERREIRA, L.C. The research on human dimensions of global environmental change in Latin America: Looking back, moving forward. International Journal of Climate Change Strategies and Management, v. 2, p. 264-280, 2010.

MCGRANAHAN, G.; BALK, D.; ANDERSON, B. The rising tide: assessing the risks of climate change and human settlements in low elevation coastal zones. Environment and Urbanization, v. 19, n. 1, p. 1737, 2007.

MENDONCA, F.A.; LEITÃO, S.A.M. Riscos e vulnerabilidade socioambiental urbana: Uma perspectiva a partir dos recursos hídricos. Geotextos (UFBA), v. 4, p. 145-163, 2008.

MENDONCA, F.A. Riscos e vulnerabilidade soicoambientais urbanos: A contingencia climática. Mercator (UFC), v. 9, p. 153-163, 2010.

MENDONCA, F.A. Riscos, Vulnerabilidades e Resiliência Sócioambientais Urbanas: Inovações na Análise Geográfica. Revista da ANPEGE, v. 7, p. 99-109, 2011.

MORGAN, M.G.; HENRION, M. Uncertainty: A Guide to Dealing with Uncertainty in Quantitative Risk and Policy Analysis. Cambridge University Press, 1990.

MOSER, C. The Asset Vulnerability Framework: Reassessing Urban Poverty Reduction Strategies. World Development, v. 26, n. 1, p. 1-19, 1998.

NICHOLLS, R.J.; HOOZEMANS, F.M.J.; MARCHAND, M. Increasing flood risk and wetland losses due to sea-level rise: regional and global analyses. Global Environmental Change, v. 9, p. S69-S87, 1999. 
NRC (National Research Council) Human Dimensions of Global Environmental Change: Research Pathways for the Next Decade. Washington DC: National Academy Press, 1999.

PARRY, M.L. Climate change is a development issue, and only sustainable development can confront the challenge. Climate and Development, v. 1, n. 1, p. 5-9, 2009.

PARRY, M.L. PALUTIKOF, J.; HANSON, C.; LOWE, J. Squaring up to reality. Nature Reports Climate Change, v. 2, p. 1-3, 2008.

PELLING, M. The Vulnerability of Cities: Natural Disasters and Social Resilience. New York: Earthscan, 2003.

PELLING, M. Adaptation to Climate Change: From Resilience to Transformation. New York: Routledge, 2010.

RAHMSTORF, S.; CAZENAVE, A.; CHURCH, J.A.; HANSEN, J.E.; KEELING, R.F.; PARKER, D.E.; SOMERVILLE, R.C.J. Recent Climate Observations Compared to Projections. Science, v. 316, p. 709, 2007.

SMIT, B.; WANDEL, J. Adaptation, adaptive capacity and vulnerability. Global Environmental Change, v. 16, p. 282-292, 2006.

SMITH, K. Environmental Hazards. London: Routledge, 1996.

SPAARGAREN, G.; MOL, A.; BUTTEL, F.H. The Environment and Global Modernity. New York: SAGE, 2000.

STENCHION, P. Development and disaster management. Australian Journal of Emergency Management, v. 12, n. 3, p. 40-44, 1997.

VEYRET, Y. Os Riscos: o Homem como agressor e vítima do meio ambiente. São Paulo: Ed. Contexto, 2007. UNDHA (United Nations Department of Humanitarian Affairs). Internationally Agreed Glossary for Basic Terms Related to Disaster Management. UNDHA, Geneve, 1992.

WILBANKS, T.J.; LANKAO, P.R.; BAO, M.; BERKHOUT, F.; CAIRNCROSS, S.; CERON, J.-P.; KAPSHE, M.; MUIR-WOOD, R.; ZAPATA-MARTI, R. Industry, settlement and society. In: PARRY, M. et al. (Orgs.) Climate Change 2007: Impacts, Adaptation and Vulnerability. Contribution of Working Group II to the Fourth Assessment Report of the Intergovernmental Panel on Climate Change. Cambridge: Cambridge University Press, p.357-390, 2007.

WISNER, B.; Blaikie, P.M.; CANNON, T.; DAVIS, I. At Risk: Natural Hazards, People's Vulnerability and Disasters. 2nd ed. London: Routledge, 2004.

Trabalho enviado em setembro de 2012

Trabalho aceito em outubro de 2012 